\title{
Integration of Systemic and Liver-Directed Therapies for Locally Advanced Hepatocellular Cancer: Harnessing Potential Synergy for New Therapeutic Horizons
}

Eric H. Bent, MD, $\mathrm{PhD}^{1}$; Eric Wehrenberg-Klee, MD²; Eugene J. Koay, MD, PhD³ ${ }^{3}$ Lipika Goyal, MD ${ }^{4, *}$; and Jennifer $\mathrm{Y}$. Wo, MD ${ }^{1, *}$

\begin{abstract}
Treatment options in locally advanced hepatocellular carcinoma (HCC) have evolved considerably over the past few years with the recent approval of multiple systemic therapies and significant advances in locoregional therapy. Given the poor prognosis for patients with unresectable $\mathrm{HCC}$, there is significant interest in rationally designed combination therapies. This article reviews the treatment options available to patients with locally advanced HCC and discusses the rationale, ongoing trials, and future prospects for combining locoregional and systemic therapy in both the definitive and neoadjuvant settings.
\end{abstract}

J Natl Compr Canc Netw 2021;19(5):567-576 doi: 10.6004/jnccn.2021.7037
Hepatocellular carcinoma (HCC) is the fourth leading cause of cancer death and the sixth most common cancer type worldwide. ${ }^{1}$ In 2020, there were an estimated 42,810 new patients with liver cancers and 30,160 deaths from liver cancers in the United States, of which HCC makes up the vast majority. ${ }^{2}$ Unfortunately, the prognosis for HCC remains poor, with 5-year survival of only $18 \%,{ }^{1,2}$ among the worst of any malignancy worldwide.

HCC is a heterogeneous disease, with variable outcomes depending on tumor stage. Curative-intent surgical resection, transplantation, or ablation are recommended for patients with early-stage tumors, defined by the Barcelona Clinic Liver Cancer (BCLC) system as stage 0 or A. For locally advanced, multifocal, or unresectable HCC disease (BCLC stage B), locoregional therapies (LRTs) including transarterial chemoembolization (TACE), transarterial radioembolization (TARE), and externalbeam radiation therapy (EBRT) are commonly considered. For patients with BCLC stage C HCC, defined by macrovascular invasion and/or distant metastases, systemic therapy is the historical standard of care. However, for liver-confined BCLC stage C disease or limited extrahepatic spread, LRT can be effective as well, with the potential to mitigate the toxicities of systemic therapy.

Systemic therapy options for unresectable or metastatic HCC have evolved considerably since 2007, when sorafenib was the only FDA-approved therapy, to the current day, wherein 9 systemic therapy regimens have full or accelerated FDA approval. Atezolizumab combined with bevacizumab is now a standard first-line treatment for unresectable or metastatic HCC based on the landmark IMbrave150 study showing the superiority of this regimen over sorafenib. ${ }^{3,4}$ Sorafenib and lenvatinib each remain standard first-line options, and multiple other vascular endothelial growth factor (VEGF)-directed

See JNCCN.org for supplemental online content. 
therapies and immunotherapies represent options in subsequent lines. Liver function and comorbidities are important considerations for patients with more advanced disease, and most of the above agents have been evaluated primarily in patients with Child-Pugh A liver function. Therapeutic options are limited for patients with significant hepatic dysfunction, including those with Child-Pugh B or C liver disease.

Multidisciplinary evaluation for patients with HCC has become essential as systemic and LRT options have expanded and evolved. In the treatment of patients with BCLC stage B and C disease, both local and systemic therapies are used depending on a variety of factors, including disease distribution, underlying liver function, comorbidities, and the expertise of the treating center..$^{5-7}$ Despite recent progress, however, prognosis remains poor. The combination of systemic therapy and LRT in this patient group represents a promising approach to improve outcomes.

In this review, we discuss the preclinical rationale and emerging clinical evidence for combining locoregional and systemic therapies in BCLC stage B and C HCC, including the potential of neoadjuvant combination therapy to improve the downstaging of tumors for surgical resection.

\section{Locoregional Therapies}

LRTs are frequently used in patients with BCLC stage B or C HCC. TACE has historically been the standard of care for most patients with BCLC stage B disease, ${ }^{8}$ but TARE and ablative EBRT, also known as stereotactic body RT (SBRT), are emerging treatment options in patients with unresectable disease. Both TACE and TARE are more frequently used in multifocal disease, whereas SBRT is more often used for large solitary tumors with or without vascular invasion.

Conventional TACE has been the standard of care for intermediate-stage BCLC stage B HCC for many years based on 2 randomized controlled trials showing a survival benefit over best supportive care. ${ }^{8-10}$ Drug-eluting bead TACE has more recently been adopted by many centers based on the results of the PRECISION V trial, which showed fewer adverse events compared with conventional TACE although no significant improvement in overall survival (OS). ${ }^{11}$

TARE is an emerging treatment approach in these patients, and retrospective data and a small phase II prospective randomized trial have shown that TARE yields improved local control with less toxicity than TACE, although without significant improvement in OS., ${ }^{5,12}$ Improved modeling and targeting of the radiation dose delivered to the tumor by TARE may further enhance disease control. ${ }^{13}$ TARE with resin beads has been compared with sorafenib in 2 phase III trials in BCLC stage B and C
HCC without distant metastases. Both of these trials included some patients with portal vein thrombus. The SARAH study, conducted in France, and the SIRveNIB study, conducted in the Asia-Pacific region, both showed a significantly higher overall response rate for TARE but similar disease control and similar OS, their primary endpoint. ${ }^{14,15}$

SBRT has also shown promising efficacy in the management of locally advanced HCC, with local control rates ranging from $87 \%$ to $100 \%$ at 1 year and OS in the range of $60 \%$ to $69 \%$ at 2 years in phase II studies. ${ }^{16,17}$ One recent phase II study of SBRT that included patients with tumors up to $12 \mathrm{~cm}$ and those with macrovascular invasion showed a 2-year local control rate of $94.8 \% .^{18} \mathrm{In}$ dicative of these emerging data, recent NCCN Clinical Practice Guidelines in Oncology (NCCN Guidelines) for Hepatobiliary Cancers ${ }^{19}$ and the American Association for the Study of Liver Diseases (AASLD) guidelines ${ }^{7}$ both list radiation as a liver-directed therapy option for the management of locally advanced HCC, but neither recommend one form of LRT over another. We recommend that treatment decisions for patients with BCLC stage B or C HCC be made after multidisciplinary evaluation and discussion.

Portal vein tumor thrombus (PVTT) is a relative contraindication for TACE, and systemic therapy has historically been the mainstay of treatment of BCLC stage C HCC with PVTT. ${ }^{20}$ However, both TARE and EBRT can be safely used in these patients, and emerging data indicate that the combination of RT and TACE may be superior to systemic therapy in this setting. ${ }^{6,21}$ Indeed, a recently published phase II randomized controlled trial showed a time to progression of 31.0 versus 11.7 weeks and an OS of 55.0 versus 43.0 weeks in patients with HCC and PVTT receiving TACE + EBRT versus sorafenib, respectively. ${ }^{21}$ In addition, several trials suggest that combinations of RT and TACE may offer better outcomes than either alone for appropriately selected patients. For example, a meta-analysis of 11 randomized controlled trials comparing TACE + RT versus TACE alone showed improved local control but with potentially greater toxicity, which may limit such use in patients with compromised hepatic function..$^{22}$ Combinations of TACE and RT, along with TACE and other locally ablative treatments such as radiofrequency ablation and microwave ablation, continue to be the subject of numerous ongoing clinical trials.

\section{Systemic Therapy}

Sorafenib, a multikinase inhibitor with activity against the VEGF, platelet-derived growth factor, and Raf signaling pathways, became the first systemic therapy to gain FDA approval for the management of unresectable or metastatic HCC in 2007. This approval was based on the SHARP trial, which showed that sorafenib yielded an 
improvement in median OS of 10.7 vs 7.9 months for placebo (hazard ratio [HR], 0.69; 95\% CI, 0.55-0.87). ${ }^{23}$ More recently, lenvatinib, a multityrosine kinase inhibitor with activity against VEGF, platelet-derived growth factor, fibroblast growth factor, Ret, and KIT and with a similar toxicity profile to sorafenib, was shown to be noninferior to sorafenib, with a median OS of 13.6 versus 12.3 months (HR, 0.92; 95\% CI, 0.79-1.06) ${ }^{24}$ and an objective response rate (ORR) of $19 \%$ versus $7 \%$, respectively. Lenvatinib received FDA approval for unresectable HCC in the first-line setting in 2018. Multiple additional single-agent targeted therapies, including regorafenib, ramucirumab, and cabozantinib, have been approved in the post-sorafenib setting, as have the PD-1 inhibitors pembrolizumab and nivolumab. ${ }^{25}$ Nivolumab and pembrolizumab showed promising response rates of $14 \%$ and
$17 \%$, respectively, in the post-sorafenib setting in the CheckMate 040 and KEYNOTE-240 trials, giving immunotherapy its first major foothold in HCC. ${ }^{25}$ Combinations of immune checkpoint inhibitors (ICIs) have demonstrated higher response rates than single agents, with nivolumab in combination with the CTLA-4 inhibitor ipilimumab showing an ORR of $32 \%$ in the secondline setting, suggesting a promising route forward in treating this disease. ${ }^{26}$

In May 2020, a combination of the PD-L1 inhibitor atezolizumab and the anti-VEGF-A antibody bevacizumab was approved for the first-line treatment of unresectable or metastatic HCC based on the results of the IMbrave150 study. ${ }^{3,4}$ This combination showed an ORR of $29.8 \%$ versus $11.3 \%$ and a median OS of 19.2 versus 13.4 months compared with sorafenib, respectively. ${ }^{25,26}$

\section{Table 1. Ongoing Prospective Trials Combining RT and Systemic Therapies}

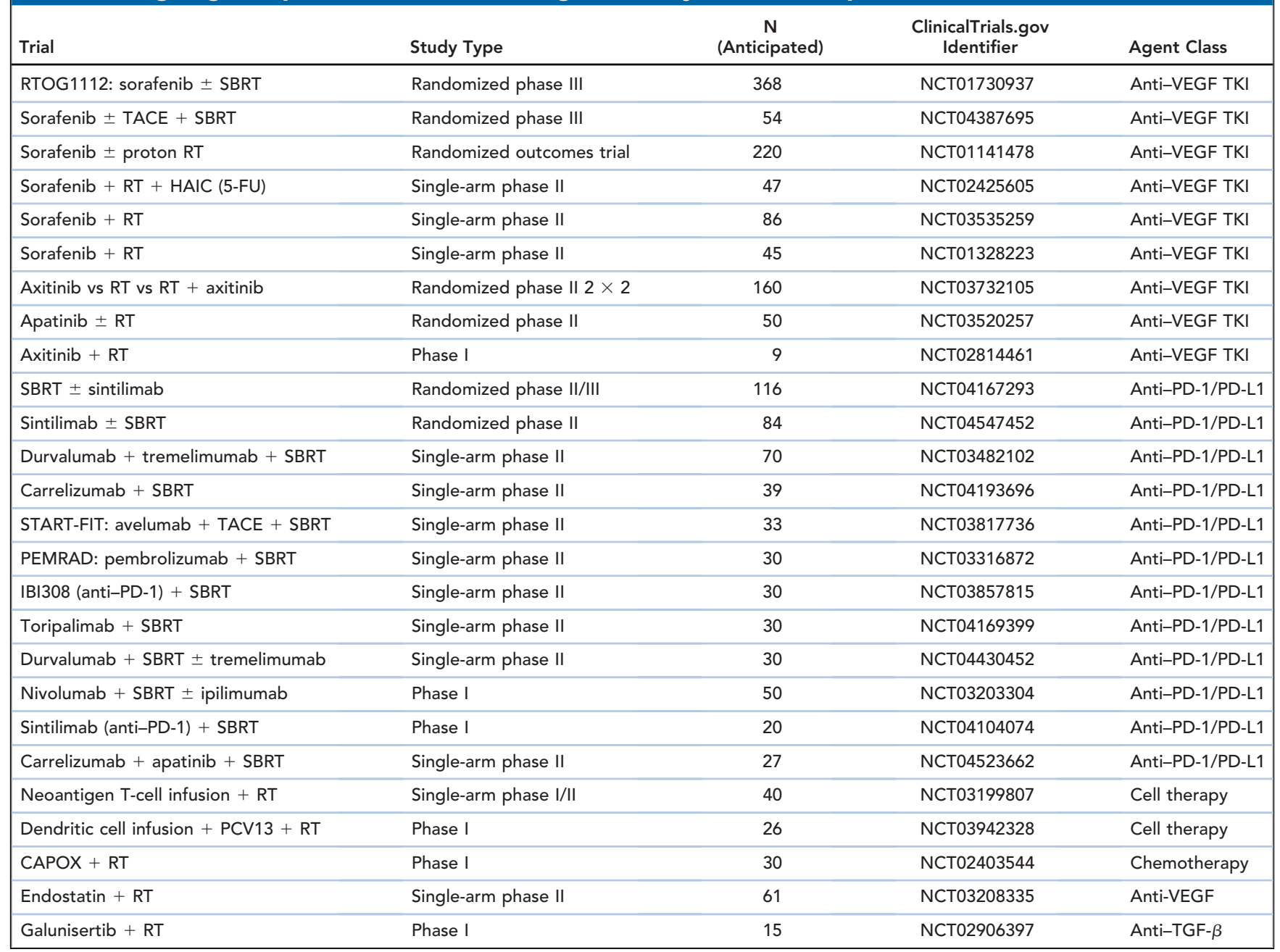

Abbreviations: CAPOX, capecitabine/oxaliplatin; HAIC, hepatic arterial infusion chemotherapy; PCV13, pneumococcal 13-valent conjugate vaccine; RT, radiation therapy; SBRT, stereotactic body radiation therapy; TACE, transarterial chemoembolization; TGF- $\beta$, transforming growth factor $\beta$; TKI, tyrosine kinase inhibitor; VEGF, vascular endothelial growth factor. 
Notably, the safety and tolerability of the combination therapy were better than those of sorafenib. These results have ushered in a new era of immunotherapy and antiVEGF combinations in HCC and establish a new standard of care. Despite these recent advances in systemic therapy, most patients with advanced HCC still experience disease progression within a year of starting therapy. Given this finding, the NCCN Guidelines emphasize that patients first be considered for enrollment on a clinical trial because the optimal immunotherapy combinations remain uncertain. There is also increasing interest in the potential of combinations of systemic therapy and LRT to enhance disease control in patients with BCLC stage B and C HCC. ${ }^{19}$

\section{Combinations of LRT With Angiogenesis Inhibitors}

Since sorafenib was approved for the treatment of unresectable HCC in 2007, there has been considerable investigation into the combination of LRTs and angiogenesis inhibitors. LRTs provide increased local disease control, whereas systemic angiogenic inhibitors can better treat microscopic disease outside the LRT treatment field. Numerous trials combining sorafenib and other VEGF targeting agents with a variety of LRTs are ongoing or completed (Tables 1-3). Sorafenib has a dual effect on HCC growth. It inhibits neoangiogenesis and tumor oxygen and nutrient supply through its activities on VEGF signaling while also blocking cancer mitogenic signaling, slowing growth, and lowering the tumor apoptotic threshold. ${ }^{27}$ This latter effect may enable enhanced tumor killing by LRT, whereas the anti-VEGF activity of sorafenib may prevent revascularization and HCC relapse after TACE by inhibiting neoangiogenesis. Sorafenib has been shown to increase radiation sensitivity both in vitro and in vivo in human HCC xenograft models, at least in part through the inhibition of VEGF-mediated ERK signaling. ${ }^{28}$ In addition, sorafenib and other VEGF inhibitors can promote the transient "normalization" of dysfunctional intratumoral blood vessels, paradoxically increasing blood flow and oxygen delivery to poorly perfused regions of the tumor while simultaneously inhibiting new blood vessel formation in the longer term. ${ }^{27}$ Vascular normalization can increase drug delivery to poorly perfused tumor regions, enhancing the efficacy of TACE and TARE, and reduce tumor hypoxia, enabling increased free-radical generation by radiation.

Although there is a promising preclinical rationale for combining LRTs with angiogenesis inhibitors, clinical results thus far have been mixed. The combination of TACE with sorafenib in unresectable HCC has shown improvement in median progression-free survival compared with TACE alone,$^{29}$ and sorafenib combined with a hepatic arterial infusion of FOLFOX (fluorouracil/leucovorin/ oxaliplatin) showed improved median OS compared with sorafenib alone in patients with HCC and PVTT, ${ }^{30}$ but most studies have failed to show an OS benefit with the combination. ${ }^{31,32}$ In the case of TARE, a phase II study of glass-bead TARE with or without sorafenib in patients with unresectable HCC actually showed a significant survival benefit for TARE alone over the combination of TARE and sorafenib. ${ }^{33}$ In addition, the recent phase III SORAMIC trial comparing the combination of resin-bead TARE and sorafenib versus sorafenib alone showed that the combination did not improve median OS. ${ }^{34}$ The large phase III RTOG 1112 study evaluating the combination of sorafenib and SBRT in patients with locally advanced HCC has recently closed to accrual, but results are not yet available (ClinicalTrials.gov identifier: NCT01730937).

\section{Biological Rationale for Combining LRT and ICls ( \pm Angiogenesis Inhibitors)}

The recent approval of multiple ICIs in HCC has raised significant interest in exploring combinations of these agents with LRTs in locally advanced HCC. Emerging preclinical and clinical data suggest that these

Table 2. Ongoing Prospective Trials Combining TARE and Systemic Therapies

\begin{tabular}{|lllll|}
\hline Trial & Study Type & N & $\begin{array}{c}\text { ClinicalTrials.gov } \\
\text { Identifier }\end{array}$ & Agent Class \\
\hline Sorafenib + TARE & Single-arm phase II & 40 & NCT01900002 & Anti-VEGF TKI \\
\hline Nivolumab + TARE & Single-arm phase II & 40 & NCT03380130 & Anti-PD-1/PD-L1 \\
\hline Nivolumab + TARE & Single-arm phase II & 40 & NCT03033446 & Anti-PD-1/PD-L1 \\
\hline Durvalumab + tremelimumab + TARE & Phase Ib & 32 & NCT04605731 & Anti-PD-1/PD-L1 \\
\hline Pembrolizumab + TARE & Early phase I & 30 & NCT03099564 & Anti-PD-1/PD-L1 \\
\hline Nivolumab + TARE & Phase I/Ib & 27 & NCT02837029 & Anti-PD-1/PD-L1 \\
\hline Durvalumab + TARE & Single-arm phase I/II & 24 & NCT04124991 & Anti-PD-1/PD-L1 \\
\hline Nivolumab + TARE & Early phase I & 2 & NCT03812562 & Anti-PD-1/PD-L1 \\
\hline
\end{tabular}

Abbreviations: TARE, transarterial radioembolization; TKI, tyrosine kinase inhibitor; VEGF, vascular endothelial growth factor. 


\section{Table 3. Selected Prospective Trials Combining TACE and Systemic Therapies}

\begin{tabular}{|c|c|c|c|c|}
\hline Trial & Study Type & $\mathbf{N}$ & $\begin{array}{l}\text { ClinicalTrials.gov } \\
\text { Identifier }\end{array}$ & Agent Class \\
\hline EMERALD-1: durvalumab \pm bevacizumab \pm TACE & Randomized phase III & 600 & NCT03778957 & Anti-PD-1/PD-L1 \\
\hline CheckMate 74W: TACE \pm nivolumab \pm ipilimumab & Randomized phase III & 765 & NCT04340193 & Anti-PD-1/PD-L1 \\
\hline LEAP-012: TACE \pm pembrolizumab + lenvatinib & Randomized phase III & 950 & NCT04246177 & Anti-PD-1/PD-L1 \\
\hline TACE-3: TACE \pm nivolumab & Randomized phase II/III & 522 & NCT04268888 & Anti-PD-1/PD-L1 \\
\hline IMMUTACE: nivolumab + TACE & Phase II & 49 & NCT03572582 & Anti-PD-1/PD-L1 \\
\hline Nivolumab + TACE & Phase I & 14 & NCT03143270 & Anti-PD-1/PD-L1 \\
\hline rAd-p53 vs TACE & Randomized phase II & 120 & NCT02418988 & Gene therapy \\
\hline TACE \pm iNKT cell infusion & Randomized phase II/III & 144 & NCT04011033 & Cell therapy \\
\hline IVY02: TACE \pm autologous immune killer cells & Randomized phase II/III & 60 & NCT03592706 & Cell therapy \\
\hline TACE \pm arginine hydrochloride \pm trimetazidine hydrochloride & Randomized phase III & 300 & NCT03274427 & Metabolic agent \\
\hline TACE \pm sorafenib & Randomized phase III & 458 & NCT00494299 & Anti-VEGF TKI \\
\hline Sorafenib \pm TACE & Randomized phase II/III & 246 & NCT01906216 & Anti-VEGF TKI \\
\hline TACE \pm sorafenib & Randomized phase III & 162 & NCT04103398 & Anti-VEGF TKI \\
\hline Sorafenib \pm TACE & Randomized phase III & 158 & NCT04143191 & Anti-VEGF TKI \\
\hline Sorafenib \pm TACE & Randomized phase III & 120 & NCT01833299 & Anti-VEGF TKI \\
\hline Apatinib + TACE & Single-arm phase II & 22 & NCT03510416 & Anti-VEGF TKI \\
\hline TACE-2: TACE \pm sorafenib & Randomized phase III & 412 & NCT01324076 & Anti-VEGF TKI \\
\hline PRODIGE16: TACE \pm sunitinib & Randomized phase III & 78 & NCT01164202 & Anti-VEGF TKI \\
\hline STAH: sorafenib \pm TACE & Randomized phase III & 339 & NCT01829035 & Anti-VEGF TKI \\
\hline
\end{tabular}

Abbreviations: iNKT, invariant natural killer T cell; TACE, transarterial chemoembolization; TKI, tyrosine kinase inhibitor; VEGF, vascular endothelial growth factor.

combinations can enhance response compared with either approach alone. LRTs may help promote systemic antitumor immunity through the induction of immunogenic cell death, resulting in the release of tumor antigens and inflammatory intracellular contents, the latter of which can increase innate immune recognition of tumor cells and promote activation of the adaptive immune system (Figure 1). Increased immune recognition of the tumor synergizes with checkpoint inhibition, which helps maintain the anticancer immune response. There may be further synergy with the addition of antiangiogenic therapy to these combinations, which has been found to promote the antitumor immune response through multiple mechanisms. . $^{25,35}$

The potential of LRTs to enhance anticancer immune responses has long been hypothesized based on the observation of abscopal responses after EBRT, in which tumors outside the radiation field shrink after irradiation of a distant site. ${ }^{36}$ Abscopal responses with local therapy are seen in HCC, although they are rare and mainly limited to case reports in the literature. ${ }^{37}$ The mechanism behind this phenomenon is thought to be immune-mediated, ${ }^{38,39}$ and radiation has been shown to activate systemic anticancer immune responses in both patients and preclinical models. ${ }^{38-42}$

TARE has also been shown to induce systemic immune activation, with increased tumor necrosis factor- $\alpha$ on circulating $\mathrm{CD}^{+}$and $\mathrm{CD} 4^{+} \mathrm{T}$ cells and a $\mathrm{CD} 8^{+} \mathrm{T}$-cell signature correlated with response. ${ }^{43}$ Cell death induced by embolization and/or the cytotoxic chemotherapy used in TACE is also immunogenic. Indeed, serum levels of immunogenic cell death biomarkers can predict TACE response ${ }^{44}$ and TACE has been shown to increase cytotoxic $\mathrm{CD}^{+}{ }^{+} \mathrm{T}$-cell and T-helper 17 cell activity and to decrease T-regulatory cell activity in the tumor microenvironment. ${ }^{45,46}$ Although thermal ablation is not often used in the BCLC stage B or C disease setting, it has also been shown to be highly immunogenic. ${ }^{47,48}$

RT and other LRTs activate anticancer immunity by inducing immunogenic tumor cell death, which involves 


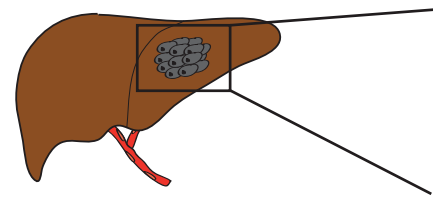

Anti-VEGF therapy Immune checkpoint blockade

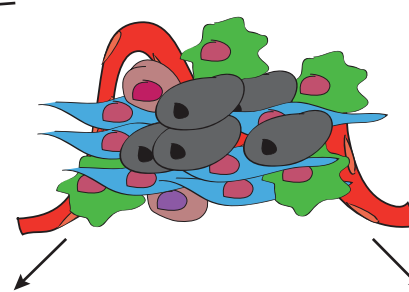

\section{EBRT}

TACE/TARE

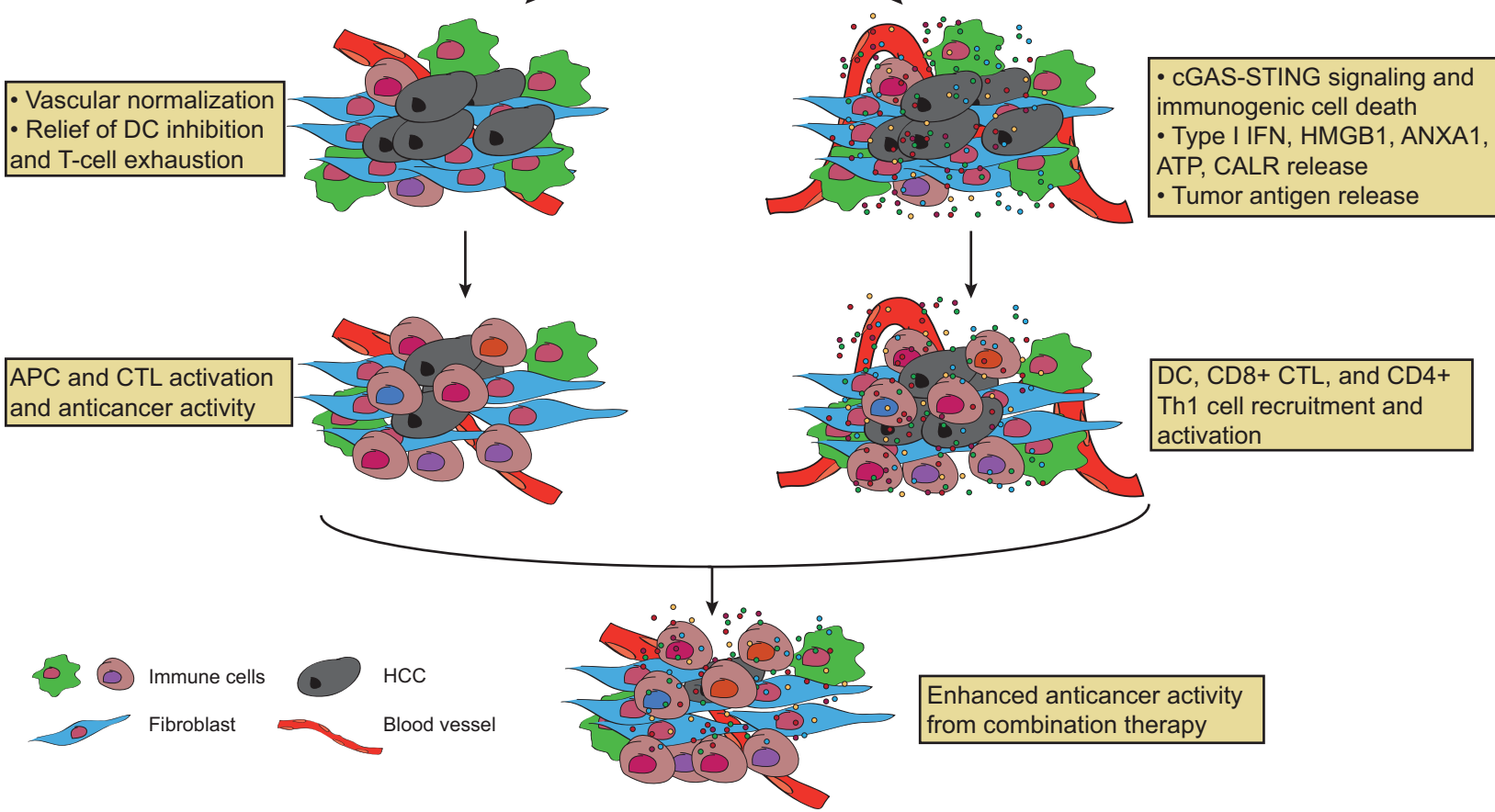

Figure 1. Schematic of LRT/immunotherapy combination rationale. Left: Anti-VEGF therapy in combination with immune checkpoint blockade (such as with the combination of atezolizumab + bevacizumab). VEGF inhibition leads to vascular normalization and increased DC and T-cell activity, enhancing the efficacy of immune checkpoint blocking antibodies. Right: Radiation and TACE lead to cGAS-STING pathway activation and immunogenic cell death with the subsequent release of a variety of cytokines and damage-associated molecular patterns, stimulating immune cell recruitment and activity. The combination of these agents may further enhance anticancer activity of each individual agent.

Abbreviations: ANXA1, annexin A1; APC, antigen-presenting cell; CALR, calreticulin; cGAS, cyclic GMP-AMP synthase; CTL, cytotoxic T-lymphocyte; DC, dendritic cell; EBRT, external-beam radiation therapy; HCC, hepatocellular carcinoma; HMGB1, high-mobility group box 1; IFN, interferon; LRT, locoregional therapy; STING, stimulator of interferon genes; TACE, transarterial chemoembolization; TARE, transarterial radioembolization; Th1, Thelper type 1 cell; VEGF, vascular endothelial growth factor.

the release of HMGB1, adenosine triphosphate, and ANXAl, among other factors, and increased surface expression of "eat-me" signals, such as calreticulin, on dying cancer cells. These signals stimulate antigen-presenting cell recruitment, activation, and tumor cell uptake, promoting the initial generation of an anticancer immune response. ${ }^{35}$ Both RT and cytotoxic chemotherapy also cause the release of DNA and RNA into the cytoplasm of damaged tumor cells, triggering cGAS-STING and Toll-like receptor signaling and subsequent inflammatory cytokine and chemokine release into the tumor microenvironment. ${ }^{49}$ In preclinical mouse models, cytosolic DNA and cGASSTING activation promotes type I interferon production and the recruitment and activation of Batf3-dependent dendritic cells and cytotoxic $\mathrm{CD}^{+} \mathrm{T}$ lymphocytes in the tumor and draining lymph nodes, stimulating anticancer immunity. ${ }^{39,49-51}$
It is thought that the addition of ICIs will help maintain this LRT-mediated antitumoral immune response, which may synergize with checkpoint inhibition. A preclinical combination of LRT with ICIs has shown impressive antitumor immunity in a number of tumor types. In HCC, the combination of radiation with CTLA-4 and PD-1 blockade can markedly reduce tumor growth in preclinical models. ${ }^{52-54}$ Similarly, the addition of cryoablation to CTLA-4 therapy has been shown to slow growth and induces immune rejection of subsequent tumors. ${ }^{55}$

Early clinical data in other malignancies combining RT with checkpoint inhibition do, in some contexts, support the rationale for combining LRT with systemic immunotherapies in HCC. An analysis of 2 recent randomized phase II clinical trials in metastatic non-small cell lung cancer showed that the addition of RT to 
pembrolizumab increased response duration, even at nonirradiated lesions, with an improvement in OS from 8.7 months with pembrolizumab alone to 19.2 months with pembrolizumab $+\mathrm{RT} .^{56}$ In contrast, in metastatic head and neck cancer, a recent phase II study of nivolumab \pm SBRT to a single metastatic lesion failed to show improved OS or response rates at nonirradiated lesions. ${ }^{57}$ The tumor type, clinical setting, and proper sequencing of these LRT/immunotherapy combinations remains the subject of significant study.

The approval of atezolizumab and bevacizumab for HCC and the promising efficacy of other immunotherapy/VEGF inhibitor combinations, including the combination of pembrolizumab and lenvatinib and of nivolumab, ipilimumab, and cabozantinib, ${ }^{58,59}$ suggest the potential of these systemic combinations to be paired with LRT and indicate the potential immunomodulatory effects of VEGF blockade. VEGF blockade can enhance anticancer immunity through a variety of mechanisms, including normalizing dysfunctional blood vessels, increasing immune cell migration into tumors, and reducing hypoxia-mediated immune suppression, and through the prevention of direct VEGF-mediated dendritic cell dysfunction and inhibition of cytotoxic T-cell function. ${ }^{25,60}$ In turn, resistance to antiangiogenic therapies may be mediated in part by an immune-suppressive microenvironment characterized by higher T-regulatory cell infiltration and stronger PD-L1 expression, ${ }^{61,62}$ arguing for the benefit of PD-1/L1 axis inhibition. Indeed, in preclinical models of HCC, lenvatinib increased tumor infiltration by $\mathrm{CD} 8^{+} \mathrm{T}$ cells and enhanced the antitumor effect of PD-1 inhibition. ${ }^{63}$ This preclinical evidence supports further clinical investigation into LRT/immunotherapy combinations in HCC.

\section{Clinical Combinations of LRT and ICls ( \pm Angiogenesis Inhibitors)}

To date, case series and single-arm studies combining LRT with ICIs in HCC have continued to suggest possible synergistic effects. A case series of 5 patients with locally advanced HCC who received SBRT and the PD-1 inhibitor nivolumab showed both safety and durable (100\%) disease control at 1 year. ${ }^{64}$ A phase I study of 14 patients with advanced HCC who received SBRT followed by nivolumab with or without the CTLA-4 inhibitor ipilimumab showed fair tolerability in both arms and preliminary efficacy. At a median follow-up of 8.3 months, the authors observed $72 \%$ disease control $(50 \%$ in the nivolumabalone arm and $87.5 \%$ in the ipilimumab/nivolumab arm). The ORR in the SBRT/ipilimumab/nivolumab arm was $50 \%$, with all of the responding patients showing durable responses lasting $>18$ months (range, 18.2-27.3 months). ${ }^{65}$ Many trials of RT and immunotherapy are ongoing, including a phase II/III trial of
SBRT and sintilimab (ClinicalTrials.gov identifier: NCT04167293) and phase II trials combining SBRT with durvalumab and tremelimumab (NCT03482102), carelizumab (NCT04193696), toripalimab (NCT04169399), and pembrolizumab (NCT03316872), among other agents (Table 1). A study in 21 patients with BCLC stage C HCC combined the CLTA- 4 inhibitor tremelimumab with local ablative therapy (10 treated with radiofrequency ablation and 11 with chemoembolization). Of patients with lesions outside the treatment zone, $26 \%$ achieved a partial response, with a median time-toprogression of 7.4 months and significant increases in $\mathrm{CD}^{+} \mathrm{T}$ cells seen in responders at 6 weeks posttreatment. ${ }^{66}$ Although these combinations have shown promise, caution must be exercised whenever novel agents are combined before prospective safety data are available. For example, increased luminal gastrointestinal toxicity has been reported when anti-VEGF agents were combined with RT. ${ }^{67-69}$ However, increased gastrointestinal toxicity has not been seen consistently in other contexts, ${ }^{70,71}$ suggesting the need for well-designed, prospective studies to establish the toxicity of novel combination therapies.

A number of ongoing studies are investigating the combination of intra-arterial therapies and ICIs, including the phase III CheckMate 74W study of TACE with nivolumab and ipilimumab (ClinicalTrials.gov identifier: NCT04340193) and phase II studies investigating TARE with nivolumab (NCT03033446, NCT03380130), TARE with pembrolizumab (NCT03099564), and TARE with durvalumab (NCT04124991), with additional studies planned (Tables 2 and 3 ).

Because of the positive results of the IMbrave150 study, ${ }^{3,4}$ significant clinical interest has been raised in exploring the addition of LRT to ICI and angiogenesis inhibitor combinations. To this end, 2 large randomized phase III trials of TACE in combination with dual VEGF and PD-1 inhibition are ongoing, including EMERALD-1 (durvalumab/bevacizumab; ClinicalTrials.gov identifier: NCT03778957) and LEAP-012 (pembrolizumab/lenvatinib; NCT04246177) (Table 3).

Ongoing LRT and systemic therapy trials are listed in Tables 1-3.

\section{Neoadjuvant Combination Therapy}

When feasible, surgery offers the best outcomes of any available treatment modality for HCC, with 5-year survival rates of $60 \%$ to $80 \%{ }^{5}$ However, many patients with BCLC stage B or C disease are not surgical candidates because of comorbid cirrhosis or high tumor burden. Furthermore, those with large, multifocal tumors or with PVTT who undergo resection have a worse prognosis. ${ }^{5,72}$ Unfortunately, tumor recurrence from either intrahepatic metastases or de novo tumor development complicates 
as many as $75 \%$ of resections at 5 years, ${ }^{73}$ and several neoadjuvant and adjuvant approaches have been tested in this setting with the hope of reducing these recurrence rates. Cytotoxic chemotherapy, TACE, and sorafenib have individually been evaluated in the neoadjuvant and adjuvant settings with mixed results. ${ }^{74-77}$ At this time, the guidelines of the AASLD and the European Association for the Study of the Liver do not recommend neoadjuvant or adjuvant therapy in most patients with resectable HCC outside of a clinical trial, but significant interest remains in these strategies. A number of large trials of immunotherapy in the adjuvant setting postresection are ongoing, including CheckMate 9DX (nivolumab; ClinicalTrials.gov identifier: NCT03383458), EMERALD-2 (durvalumab/bevacizumab; NCT03847428), JUPITER-04 (toripalimab; NCT03859128), and KEYNOTE-937 (pembrolizumab; NCT03867084).

Of particular and emerging interest in the treatment of borderline or unresectable BCLC stage B or C HCC is the potential of neoadjuvant therapies to downstage patients. Early data from a phase II trial of neoadjuvant nivolumab versus nivolumab combined with ipilimumab showed pathologic complete response rates of $25 \%$, suggesting that this approach may enable some patients with locally advanced disease to successfully proceed to surgery. ${ }^{78}$ These data, and the results of the IMbrave150 study, which showed an ORR of $29.8 \%$ with $7.7 \%$ complete response in patients receiving atezolizumab and bevacizumab, ${ }^{3,4}$ raise the prospect that both LRT and immunotherapy, either alone or in combination, may be useful in downstaging unresectable HCC to enable curative-intent resection. Although clinical outcomes from most studies investigating immunotherapy and LRT in the neoadjuvant setting are pending, case reports provide a proof of concept that immunotherapy combinations can downstage patients with unresectable HCC. ${ }^{79}$ Of particular promise are studies that combine ICIs and LRT in the neoadjuvant setting. A recent case series of 5 patients with locally advanced HCC treated using SBRT and checkpoint inhibition reported $40 \%$ complete response, with $39 \%$ median tumor shrinkage and 1 patient proceeding to curative-intent radiofrequency ablation. ${ }^{64}$ Based on these findings, the START-FIT trial (ClinicalTrials.gov identifier: NCT03817736) has been launched to evaluate the sequential combination of TACE, hypofractionated SBRT, and the ICI avelumab to downstage locally advanced HCC before hepatectomy.

Although the role of surgery is controversial in the setting of HCC with PVTT, data support the role of neoadjuvant RT before resection in this population. In a recent randomized multicenter study of patients with resectable HCC and PVTT, neoadjuvant RT provided significantly increased disease-free survival and OS compared with surgery alone, with an HR for HCC-related mortality of 0.35 (95\% CI, $0.23-0.54$ ) on multivariate analysis, and OS of $27.4 \%$ versus $9.4 \%$ at 24 months, respectively, despite the use of very low radiation doses well below the ablative range. The regimen was tolerable, with minimal grade $\geq 3$ toxicity and the vast majority of patients proceeding to surgery. ${ }^{80}$ The addition of immunotherapy to neoadjuvant regimens like this may further improve these promising initial results. As improved systemic therapy and LRT combinations are identified in more advanced disease, the potential for these agents to successfully downstage patients with borderline resectable HCC, enabling curative therapy, should continue to be explored. We await the results of ongoing studies investigating a variety of neoadjuvant approaches (supplemental eTable 1, available with this article at JNCCN.org ).

\section{Conclusions}

The treatment landscape in HCC continues to evolve rapidly, with the combination of liver-directed therapy and systemic therapy holding real promise for patients with HCC. Preliminary correlative studies and preclinical data suggest a biological rationale for the combination of LRTs with immunotherapy and VEGF inhibitors, and emerging clinical data in patients with BCLC stage B and $\mathrm{C}$ disease reinforce the promise of this approach. Multidisciplinary evaluation of patients with HCC is increasingly critical as traditional options for treatments by stage begin to expand. Systemic therapy is now being evaluated in early-stage disease, and liver-directed therapy is being evaluated in late-stage disease; the optimal approach and setting to combine these approaches will be guided by the highly anticipated results of several ongoing studies.

Submitted December 4, 2020; accepted for publication March 16, 2021.

Disclosures: Dr. Wehrenberg-Klee has disclosed receiving consulting fees from Sirtex Medical Ltd., and grant/research support from Boston Scientific. Dr. Koay has disclosed receiving grant/research support from Philips Healthcare, General Electric, Elekta, and Stand Up 2 Cancer; and receiving personal fees from RenovoRx and Taylor \& Francis, LLC. Dr. Goyal has disclosed serving as a scientific advisor for Agios Pharmaceuticals Inc., Alentis Therapeutics AG, Debiopharm Group, H3 Biomedicine, Incyte Corporation, QED Therapeutics, Sirtex Medical Ltd., and Taiho Oncology Inc; receiving consulting fees from Agios Pharmaceuticals Inc., Alentis Therapeutics AG, Incyte Corporation, QED Therapeutics, Sirtex Medica Ltd., and Taiho Oncology Inc.; and serving as a scientific consultant for AstraZeneca Pharmaceuticals LP. Dr. Wo has disclosed receiving grant/ research support from Genentech. Dr. Bent has disclosed that he has not received any financial consideration from any person or organization to support the preparation, analysis, results, or discussion of this article.

Correspondence: Lipika Goyal, MD, Division of Hematology and Oncology, Department of Medicine, Massachusetts General Hospital, Yawkey 7E, 55 Fruit Street, Boston, MA 02114. Email: Igoyal@partners.org; and Jennifer Y. Wo, MD, Department of Radiation Oncology, Massachusetts General Hospital, 100 Blossom Street, Boston, MA 02114. Email: jwo@ partners.org 


\section{References}

1. Villanueva A. Hepatocellular carcinoma. N Engl J Med 2019;380: 1450-1462.

2. Howlader N, Noone AM, Krapcho M, et al. SEER Cancer Statistics Review, 1975-2017. Accessed March 22, 2021. Available at: https://seer. cancer.gov/csr/1975_2017/

3. Finn RS, Qin $S$, Ikeda $M$, et al. Atezolizumab plus bevacizumab in unresectable hepatocellular carcinoma. N Engl J Med 2020;382:1894-1905.

4. Finn RS, Qin S, Ikeda M, et al. IMbrave150: updated overall survival (OS) data from a global, randomized, open-label phase III study of atezolizumab (atezo) + bevacizumab (bev) versus sorafenib (sor) in patients (pts) with unresectable hepatocellular carcinoma (HCC) [abstract]. J Clin Oncol 2021;39(Suppl):Abstract 267

5. European Association for the Study of the Liver. EASL Clinical Practice Guidelines: management of hepatocellular carcinoma. J Hepatol 2018;69:182-236.

6. Cheng $\mathrm{S}$, Chen M, Cai J, et al. Chinese expert consensus on multidisciplinary diagnosis and treatment of hepatocellular carcinoma with portal vein tumor thrombus (2018 edition). Liver Cancer 2020;9:28-40.

7. Heimbach JK, Kulik LM, Finn RS, et al. AASLD guidelines for the treatment of hepatocellular carcinoma. Hepatology 2018;67:358-380.

8. Lencioni R, de Baere T, Soulen MC, et al. Lipiodol transarterial chemoembolization for hepatocellular carcinoma: a systematic review of efficacy and safety data. Hepatology 2016;64:106-116.

9. Llovet JM, Real MI, Montaña X, et al. Arterial embolisation or chemoembolisation versus symptomatic treatment in patients with unresectable hepatocellular carcinoma: a randomised controlled trial. Lancet 2002;359: 1734-1739.

10. Lo CM, Ngan H, Tso WK, et al. Randomized controlled trial of transarterial lipiodol chemoembolization for unresectable hepatocellular carcinoma. Hepatology 2002;35:1164-1171.

11. Lammer J, Malagari K, Vogl T, et al. Prospective randomized study of doxorubicin-eluting-bead embolization in the treatment of hepatocellular carcinoma: results of the PRECISION V study. Cardiovasc Intervent Radiol 2010;33:41-52.

12. Salem R, Gordon AC, Mouli S, et al. Y90 radioembolization significantly prolongs time to progression compared with chemoembolization in patients with hepatocellular carcinoma. Gastroenterology 2016;151: 1155-1163.e2.

13. O'Doherty J. A review of 3D image-based dosimetry, technical considerations and emerging perspectives in 90Y microsphere therapy. J Diagn Imaging Ther 2015;2:1-34.

14. Vilgrain $V$, Pereira $H$, Assenat $E$, et al. Efficacy and safety of selective internal radiotherapy with yttrium-90 resin microspheres compared with sorafenib in locally advanced and inoperable hepatocellular carcinoma (SARAH): an open-label randomised controlled phase 3 trial. Lancet Oncol 2017;18:1624-1636.

15. Chow PKH, Gandhi M, Tan SB, et al. SIRveNIB: selective internal radiation therapy versus sorafenib in Asia-Pacific patients with hepatocellular carcinoma. J Clin Oncol 2018;36:1913-1921.

16. Bujold A, Massey CA, Kim JJ, et al. Sequential phase I and II trials of stereotactic body radiotherapy for locally advanced hepatocellular carcinoma. J Clin Oncol 2013;31:1631-1639.

17. Andolino DL, Johnson CS, Maluccio M, et al. Stereotactic body radiotherapy for primary hepatocellular carcinoma. Int J Radiat Oncol Biol Phys 2011;81:e447-453

18. Hong TS, Wo JY, Yeap BY, et al. Multi-institutional phase II study of highdose hypofractionated proton beam therapy in patients with localized, unresectable hepatocellular carcinoma and intrahepatic cholangiocarcinoma. J Clin Oncol 2016;34:460-468.

19. Benson AB III, D'Angelica MI, Abbott DE, et al. NCCN Clinical Practice Guidelines in Oncology: Hepatobiliary Cancers. Version 2.2021. Accessed April 18, 2021. To view the most recent version, visit NCCN.org

20. Llovet JM, Brú C, Bruix J. Prognosis of hepatocellular carcinoma: the BCLC staging classification. Semin Liver Dis 1999;19:329-338.

21. Yoon SM, Ryoo BY, Lee SJ, et al. Efficacy and safety of transarterial chemoembolization plus external beam radiotherapy vs sorafenib in hepatocellular carcinoma with macroscopic vascular invasion: a randomized clinical trial. JAMA Oncol 2018;4:661-669

22. Huo YR, Eslick GD. Transcatheter arterial chemoembolization plus radiotherapy compared with chemoembolization alone for hepatocellular carcinoma: a systematic review and meta-analysis. JAMA Oncol 2015;1: 756-765.
23. Llovet JM, Ricci S, Mazzaferro V, et al. Sorafenib in advanced hepatocellular carcinoma. N Engl J Med 2008;359:378-390.

24. Kudo M, Finn RS, Qin S, et al. Lenvatinib versus sorafenib in first-line treatment of patients with unresectable hepatocellular carcinoma: a randomised phase 3 non-inferiority trial. Lancet 2018;391:1163-1173.

25. Pinter M, Jain RK, Duda DG. The current landscape of immune checkpoint blockade in hepatocellular carcinoma: a review. JAMA Oncol 2021;7:113-123.

26. Yau T, Kang YK, Kim TY, et al. Efficacy and safety of nivolumab plus ipilimumab in patients with advanced hepatocellular carcinoma previously treated with sorafenib: the CheckMate 040 randomized clinical trial. JAMA Oncol 2020;6:e204564.

27. Jain RK. Normalization of tumor vasculature: an emerging concept in antiangiogenic therapy. Science 2005;307:58-62.

28. Yu W, Gu K, Yu Z, et al. Sorafenib potentiates irradiation effect in hepatocellular carcinoma in vitro and in vivo. Cancer Lett 2013;329:109-117.

29. Kudo M, Ueshima K, lkeda M, et al. Randomised, multicentre prospective trial of transarterial chemoembolisation (TACE) plus sorafenib as compared with TACE alone in patients with hepatocellular carcinoma: TACTICS trial. Gut 2020;69:1492-1501.

30. He M, Li Q, Zou R, et al. Sorafenib plus hepatic arterial infusion of oxaliplatin, fluorouracil, and leucovorin vs sorafenib alone for hepatocellular carcinoma with portal vein invasion: a randomized clinical trial. JAMA Oncol 2019;5:953-960.

31. Meyer T, Fox R, Ma YT, et al. Sorafenib in combination with transarterial chemoembolisation in patients with unresectable hepatocellular carcinoma (TACE 2): a randomised placebo-controlled, double-blind, phase 3 trial. Lancet Gastroenterol Hepatol 2017;2:565-575.

32. Li L, Zhao W, Wang M, et al. Transarterial chemoembolization plus sorafenib for the management of unresectable hepatocellular carcinoma: a systematic review and meta-analysis. BMC Gastroenterol 2018;18:138.

33. Teyateeti A, Mahvash A, Long JP, et al. Survival outcomes for yttrium-90 transarterial radioembolization with and without sorafenib for unresectable hepatocellular carcinoma patients. J Hepatocell Carcinoma 2020;7:117-131.

34. Ricke J, Klümpen $\mathrm{HJ}$, Amthauer $\mathrm{H}$, et al. Impact of combined selective in ternal radiation therapy and sorafenib on survival in advanced hepatocellular carcinoma. J Hepatol 2019;71:1164-1174.

35. Kroemer G, Galluzzi L, Kepp O, et al. Immunogenic cell death in cancer therapy. Annu Rev Immunol 2013;31:51-72.

36. Postow MA, Callahan MK, Barker CA, et al. Immunologic correlates of the abscopal effect in a patient with melanoma. N Engl J Med 2012;366:925-931.

37. Beyls C, Haustermans K, Deroose CM, et al. Could autoimmune disease contribute to the abscopal effect in metastatic hepatocellular carcinoma? Hepatology 2020;72:1152-1154.

38. Demaria $\mathrm{S}, \mathrm{Ng} \mathrm{B}$, Devitt $\mathrm{ML}$, et al. lonizing radiation inhibition of distant untreated tumors (abscopal effect) is immune mediated. Int J Radiat Oncol Biol Phys 2004;58:862-870.

39. Rodríguez-Ruiz ME, Vanpouille-Box C, Melero I, et al. Immunological mechanisms responsible for radiation-induced abscopal effect. Trends Immunol 2018;39:644-655.

40. Formenti SC, Rudqvist NP, Golden E, et al. Radiotherapy induces responses of lung cancer to CTLA-4 blockade. Nat Med 2018;24: 1845-1851.

41. Chi KH, Liu SJ, Li CP, et al. Combination of conformal radiotherapy and intratumoral injection of adoptive dendritic cell immunotherapy in refractory hepatoma. J Immunother 2005;28:129-135.

42. Grassberger C, Hong TS, Hato T, et al. Differential association between circulating lymphocyte populations with outcome after radiation therapy in subtypes of liver cancer. Int J Radiat Oncol Biol Phys 2018;101: 1222-1225.

43. Chew $\mathrm{V}$, Lee $\mathrm{YH}$, Pan $\mathrm{L}$, et al. Immune activation underlies a sustained clinical response to yttrium-90 radioembolisation in hepatocellular carcinoma. Gut 2019;68:335-346

44. Kohles N, Nagel D, Jüngst D, et al. Predictive value of immunogenic cell death biomarkers HMGB1, sRAGE, and DNase in liver cancer patients receiving transarterial chemoembolization therapy. Tumour Biol 2012;33:2401-2409

45. Singh $\mathrm{P}$, Toom $\mathrm{S}$, Avula $\mathrm{A}$, et al. The immune modulation effect of locoregional therapies and its potential synergy with immunotherapy in hepatocellular carcinoma. J Hepatocell Carcinoma 2020;7:11-17. 
46. Hiroishi K, Eguchi J, Baba T, et al. Strong CD8(+) T-cell responses against tumor-associated antigens prolong the recurrence-free interval after tumor treatment in patients with hepatocellular carcinoma. J Gastroenterol 2010;45:451-458

47. Zerbini A, Pilli M, Laccabue D, et al. Radiofrequency thermal ablation for hepatocellular carcinoma stimulates autologous NK-cell response. Gastroenterology 2010;138:1931-1942.

48. Hansler J, Wissniowski TT, Schuppan D, et al. Activation and dramatically increased cytolytic activity of tumor specific $T$ lymphocytes after radiofrequency ablation in patients with hepatocellular carcinoma and colorectal liver metastases. World J Gastroenterol 2006;12:3716-3721.

49. Vanpouille-Box C, Alard A, Aryankalayil MJ, et al. DNA exonuclease Trex1 regulates radiotherapy-induced tumour immunogenicity. Nat Commun 2017;8:15618.

50. Weichselbaum RR, Liang H, Deng L, et al. Radiotherapy and immunotherapy: a beneficial liaison? Nat Rev Clin Oncol 2017;14:365-379.

51. Twyman-Saint Victor C, Rech AJ, Maity A, et al. Radiation and dual checkpoint blockade activate non-redundant immune mechanisms in cancer. Nature 2015;520:373-377.

52. Kim KJ, Kim JH, Lee SJ, et al. Radiation improves antitumor effect of im mune checkpoint inhibitor in murine hepatocellular carcinoma model. Oncotarget 2017;8:41242-41255.

53. Friedman D, Baird JR, Young KH, et al. Programmed cell death-1 blockade enhances response to stereotactic radiation in an orthotopic murine model of hepatocellular carcinoma. Hepatol Res 2017;47:702-714.

54. Choi C, Yoo GS, Cho WK, et al. Optimizing radiotherapy with immune checkpoint blockade in hepatocellular carcinoma. World J Gastroenterol 2019;25:2416-2429.

55. Waitz R, Solomon SB, Petre EN, et al. Potent induction of tumor immunity by combining tumor cryoablation with anti-CTLA-4 therapy. Cancer Res 2012;72:430-439.

56. Theelen WSME, Chen D, Verma V, et al. Pembrolizumab with or without radiotherapy for metastatic non-small-cell lung cancer: a pooled analysis of two randomised trials [published online October 20, 2020]. Lancet Respir Med, doi.org/10.1016/S2213-2600(20)30391-X

57. McBride S, Sherman E, Tsai CJ, et al. Randomized phase II trial of nivolumab with stereotactic body radiotherapy versus nivolumab alone in metastatic head and neck squamous cell carcinoma. J Clin Oncol 2021;39: 30-37

58. Zhu AX, Finn RS, Ikeda $M$, et al. A phase lb study of lenvatinib (LEN) plus pembrolizumab (PEMBRO) in unresectable hepatocellular carcinoma (uHCC) [abstract]. J Clin Oncol 2020;38(Suppl):Abstract 4519

59. Yau T, Zagonel V, Santoro A, et al. Nivolumab (NIVO) + ipilimumab (IPI) + cabozantinib (CABO) combination therapy in patients (pts) with advanced hepatocellular carcinoma (aHCC): results from CheckMate 040 [abstract]. J Clin Oncol 2020;38(Suppl):Abstract 478.

60. Munn LL, Jain RK. Vascular regulation of antitumor immunity. Science 2019;365:544-545.

61. Liu XD, Hoang A, Zhou L, et al. Resistance to antiangiogenic therapy is associated with an immunosuppressive tumor microenvironment in metastatic renal cell carcinoma. Cancer Immunol Res 2015;3:1017-1029.

62. Lu LC, Lee $\mathrm{YH}$, Chang CJ, et al. Increased expression of programmed death-ligand 1 in infiltrating immune cells in hepatocellular carcinoma tissues after sorafenib treatment. Liver Cancer 2019;8:110-120.

63. Kimura T, Kato Y, Ozawa $Y$, et al. Immunomodulatory activity of lenvatinib contributes to antitumor activity in the Hepa1-6 hepatocellular carcinoma model. Cancer Sci 2018;109:3993-4002.
64. Chiang $\mathrm{CL}$, Chan ACY, Chiu KWH, et al. Combined stereotactic body radiotherapy and checkpoint inhibition in unresectable hepatocellular carcinoma: a potential synergistic treatment strategy. Front Oncol 2019;9:1157.

65. Juloori A, Liao CY, Lemons JM, et al. Phase I study of stereotactic body radiotherapy followed by ipilimumab with nivolumab vs nivolumab alone in unresectable hepatocellular carcinoma. Int J Radiat Oncol Biol Phys 2020;108:S149-150

66. Duffy AG, Ulahannan SV, Makorova-Rusher O, et al. Tremelimumab in combination with ablation in patients with advanced hepatocellular carcinoma. J Hepatol 2017;66:545-551.

67. Pollom EL, Deng L, Pai RK, et al. Gastrointestinal toxicities with combined antiangiogenic and stereotactic body radiation therapy. Int J Radiat Oncol Biol Phys 2015;92:568-576.

68. Chen SW, Lin LC, Kuo YC, et al. Phase 2 study of combined sorafenib and radiation therapy in patients with advanced hepatocellular carcinoma. Int J Radiat Oncol Biol Phys 2014;88:1041-1047.

69. Spigel DR, Hainsworth JD, Yardley DA, et al. Tracheoesophageal fistula formation in patients with lung cancer treated with chemoradiation and bevacizumab. J Clin Oncol 2010;28:43-48

70. Small W Jr, Mulcahy MF, Rademaker A, et al. Phase II trial of full-dose gemcitabine and bevacizumab in combination with attenuated threedimensional conformal radiotherapy in patients with localized pancreatic cancer. Int J Radiat Oncol Biol Phys 2011;80:476-482.

71. Crane $\mathrm{CH}$, Eng $\mathrm{C}$, Feig BW, et al. Phase II trial of neoadjuvant bevacizumab, capecitabine, and radiotherapy for locally advanced rectal cancer. Int J Radiat Oncol Biol Phys 2010;76:824-830.

72. Vitale A, Burra P, Frigo AC, et al. Survival benefit of liver resection for patients with hepatocellular carcinoma across different Barcelona Clinic Liver Cancer stages: a multicentre study. J Hepatol 2015;62:617-624.

73. Pompili M, Saviano A, de Matthaeis N, et al. Long-term effectiveness of resection and radiofrequency ablation for single hepatocellular carcinoma $\leq 3 \mathrm{~cm}$. Results of a multicenter Italian survey. J Hepatol 2013;59:89-97.

74. Samuel M, Chow PK, Chan Shih-Yen E, et al. Neoadjuvant and adjuvant therapy for surgical resection of hepatocellular carcinoma. Cochrane Database Syst Rev 2009;2009:CD001199.

75. Bruix J, Takayama T, Mazzaferro V, et al. Adjuvant sorafenib for hepatocellular carcinoma after resection or ablation (STORM): a phase 3, randomised, double-blind, placebo-controlled trial. Lancet Oncol 2015;16: 1344-1354.

76. Wang J, He XD, Yao N, et al. A meta-analysis of adjuvant therapy after potentially curative treatment for hepatocellular carcinoma. Can J Gastroenterol 2013;27:351-363.

77. Wang Z, Ren Z, Chen Y, et al. Adjuvant transarterial chemoembolization for HBV-related hepatocellular carcinoma after resection: a randomized controlled study. Clin Cancer Res 2018;24:2074-2081.

78. Kaseb A, Pestana RC, Vence LM, et al. Randomized, open-label, perioperative phase II study evaluating nivolumab alone versus nivolumab plus ipilimumab in patients with resectable HCC [abstract]. J Clin Oncol 2019;37(Suppl):Abstract 185

79. Chen $X$, Zhang $Y$, Zhang $N$, et al. Lenvatinib combined nivolumab injection followed by extended right hepatectomy is a feasible treatment for patients with massive hepatocellular carcinoma: a case report. OncoTargets Ther 2019;12:7355-7359.

80. Wei $X$, Jiang $Y$, Zhang $X$, et al. Neoadjuvant three-dimensional conformal radiotherapy for resectable hepatocellular carcinoma with portal vein tumor thrombus: a randomized, open-label, multicenter controlled study. J Clin Oncol 2019;37:2141-2151. 
Supplemental online content for:

\section{Integration of Systemic and Liver-Directed Therapies for Locally Advanced Hepatocellular Cancer: Harnessing Potential Synergy for New Therapeutic Horizons}

Eric H. Bent, MD, PhD; Eric Wehrenberg-Klee, MD; Eugene J. Koay, MD, PhD; Lipika Goyal, MD; and Jennifer Y. Wo, MD

J Natl Compr Canc Netw 2021;19(5):567-576

eTable 1: Ongoing Prospective Trials of Neoadjuvant Systemic and Local Therapy 


\section{eTable 1. Ongoing Prospective Trials of Neoadjuvant Systemic and Local Therapy}

\begin{tabular}{|c|c|c|c|c|}
\hline Trial & Study Type & $\mathbf{N}$ & $\begin{array}{l}\text { ClinicalTrials.gov } \\
\text { Identifier }\end{array}$ & Agent Class \\
\hline Carrelizumab + apatinib & Randomized phase II/III & 514 & NCT04521153 & Anti-PD-1/PD-L1 + anti-VEGF TKI \\
\hline Pembrolizumab + lenvatinib & Randomized outcomes trial & 192 & NCT04425226 & Anti-PD-1/PD-L1 + anti-VEGF TKI \\
\hline Cemiplimab & Randomized phase II & 94 & NCT03916627 & Anti-PD-1/PD-L1 \\
\hline Sintilimab + TACE & Single-arm phase II & 61 & NCT04174781 & Anti-PD-1/PD-L1 + TACE \\
\hline Nivolumab \pm BMS- 813160 or BMS- 986253 & Randomized phase II & 50 & NCT04123379 & Anti-PD-1 + anti-CCR2/5 or anti-IL-8 \\
\hline Nivolumab + ipilimumab & Single-arm phase II & 40 & NCT03510871 & Anti-PD-1/PD-L1 + anti-CTLA-4 \\
\hline Nivolumab + ipilimumab & Single-arm phase II & 40 & NCT03510871 & Anti-PD-1/PD-L1 + anti-CTLA-4 \\
\hline START-FIT: SBRT + TACE + avelumab & Single-arm phase II & 33 & NCT03817736 & Anti-PD-1/PD-L1 + RT + TACE \\
\hline Durvalumab + lenvatinib & Single-arm & 20 & NCT04443322 & Anti-PD-1/PD-L1 + anti-VEGF TKI \\
\hline Nivolumab + cabozantinib & Phase I & 15 & NCT03299946 & Anti-PD-1/PD-L1 + anti-VEGF TKI \\
\hline Toripalimab & Single-arm phase $\mathrm{lb} / \mathrm{II}$ & 20 & NCT03867370 & Anti-PD-1/PD-L1 \\
\hline SHR-210 (PD-1) + apatinib & Single-arm phase II & 20 & NCT04297202 & Anti-PD-1/PD-L1 + anti-VEGF TKI \\
\hline SBRT & Randomized phase II/III & 214 & NCT04025437 & RT \\
\hline RT & Randomized phase II & 50 & NCT02580929 & RT \\
\hline SBRT & Phase I & 30 & NCT04587739 & RT \\
\hline SBRT & N/A & 35 & NCT04186234 & RT \\
\hline HAIC (mFOLFOX) & Randomized phase III & 344 & NCT03851913 & HAIC (mFOLFOX) \\
\hline HAIC (mFOLFOX) & Randomized phase III & 230 & NCT03368651 & HAIC (mFOLFOX) \\
\hline HAIC (mFOLFOX) & Randomized phase III & 40 & NCT04595864 & HAIC (mFOLFOX) \\
\hline
\end{tabular}

Abbreviations: FOLFOX, fluorouracil/leucovorin/oxaliplatin; HAIC, hepatic arterial infusion chemotherapy; IL, interleukin; mFOLFOX, modified FOLFOX; N/A not available; RT, radiation therapy; SBRT, stereotactic body radiation therapy; TACE, transarterial chemoembolization; TKI, tyrosine kinase inhibitor; VEGF, vascular endothelial growth factor. 